\title{
Wolfgang Adam, Verspätete Ankunft. Montaignes "Journal de voyage" im 18. Jahrhundert. Rezeption eines frühneuzeitlichen Textes
}

\section{Concetta Cavallini}

\section{(2) OpenEdition \\ 12 Journals}

\section{Édition électronique}

URL : https://journals.openedition.org/studifrancesi/3054

DOI : 10.4000/studifrancesi.3054

ISSN : 2421-5856

\section{Éditeur}

Rosenberg \& Sellier

\section{Édition imprimée}

Date de publication : 1 juillet 2013

Pagination : 444-445

ISSN : 0039-2944

\section{Référence électronique}

Concetta Cavallini, «Wolfgang Adam, Verspätete Ankunft. Montaignes "Journal de voyage" im 18. Jahrhundert. Rezeption eines frühneuzeitlichen Textes », Studi Francesi [En ligne], 170 (LVII | II) | 2013, mis en ligne le 30 novembre 2015, consulté le 31 janvier 2023. URL : http://journals.openedition.org/ studifrancesi/3054; DOI : https://doi.org/10.4000/studifrancesi.3054

Ce document a été généré automatiquement le 31 janvier 2023.

\section{c)}

Creative Commons - Attribution - Pas d'Utilisation Commerciale - Pas de Modification 4.0 International - CC BY-NC-ND 4.0

https://creativecommons.org/licenses/by-nc-nd/4.0/ 


\title{
Wolfgang Adam, Verspätete Ankunft. Montaignes "Journal de voyage" im 18. Jahrhundert. Rezeption eines frühneuzeitlichen Textes
}

\author{
Concetta Cavallini
}

\section{RÉFÉRENCE}

WOLFGANG ADAM, Verspätete Ankunft. Montaignes "Journal de voyage" im 18. Jahrhundert.

Rezeption eines frühneuzeitlichen Textes, Heidelberg, Universitätsverlag Winter, 2012, pp.

209.

Pour le Journal de voyage de Montaigne, l'Allemagne représente un pays d'élection: ce n'est que dans ce pays, en effet, qu'on traduisit le texte de la première édition française de 1774 déjà en 1777-1779, donc quelques années seulement après sa publication. L'édition, en deux volumes (Michael Von Montaigne. Reisen Durch die Schweiz, Deutschland und Italien, in den Jahren 1580 und 1581, aus dem Französischen, mit Zusätzen, Halle, J. C. Hendel), fut procurée par Jean-Henri-Frédéric Ulrich. L'Allemagne occupe ainsi une position privilégiée dans le panorama des traductions du Journal, surtout si l'on rappelle que la première traduction anglaise ne parut qu'en 1842 (William Hazlitt) et que la première traduction italienne n'arriva qu'un siècle après la traduction anglaise (Irene Riboni, 1942). Le volume de Wolfgang Adam, qui vient tout juste de paraître aux Presses de l'Université d'Heidelberg, comble une lacune importante dans l'histoire de la réception du Journal de voyage de Montaigne. Les attentes déterminées par la découverte de Joseph Prunis en 1770-71, et donc en pleine époque des Lumières, d'un manuscrit de Montaigne, le philosophe par excellence, furent nombreuses en France et à l'étranger. Cependant, le coup éditorial, auquel les journaux de l'époque avaient consacré beaucoup d'attention, tourna à l'échec. Les rivalités personnelles que bien des journalistes nourrissaient à l'égard de Querlon, le premier éditeur du manuscrit, ainsi 
que la nature du texte de Montaigne, un journal sec et laconique, dépourvu du souffle des Essais, contribuèrent à la mise à l'écart de l'œuvre. Mathurin Dréano, dans La renommée de Montaigne en France au XVIII siècle (Angers, Editions de l'Ouest, 1952), analyse magistralement tous les événements liés à la première réception du texte du Journal. L'étude rigoureuse de Wolfgang Adam ajoute donc des éléments fondamentaux à l'histoire critique de la réception du Journal en Europe. Le premier chapitre du volume («Faire revivre Montaigne: Die Geschichte der Auffindung und Edition des Journal de voyage», pp. 9-31) reparcourt l'histoire de la découverte du manuscrit et de la publication des premières éditions françaises. Le deuxième et le troisième chapitre, qui constituent la partie centrale et capitale de l'étude, sont presque symétriques. Ils sont consacrés aux premiers protagonistes de l'histoire éditoriale du Journal: à Meunier de Querlon, responsable de la première édition française, et à Johann Heinrich Friedrich Ulrich, premier traducteur du texte en allemand (1777-1779) ainsi que premier traducteur dans l'absolu du Journal de Montaigne. L'analyse menée sur le travail d'édition de Querlon («Die Stimme Meunier de Querlons: ein homme de lettres des 18. Jahrhunderts kommentiert Montaignes Text», pp. 33-99), descend dans le détail des choix linguistiques, philologiques et textuels accomplis par le journaliste français. Les notes culturelles ne sont pas laissées de côté, puisque leur rôle, dans la réception du texte du Journal, fut essentiel. L'ensemble du troisième chapitre est consacré à la traduction allemande de Ulrich («Montaignes Reisetagebuch in Deutsch», pp. 101-170). Le traducteur allemand est en effet une figure presque inconnue dans le domaine francophone, si ce n'est pour une note transcrite par le Dr Payen dans la copie de la traduction allemande du «Fonds Payen» (Bibliothèque nationale de France, Z-Payen 432-33): «Jean-Henri-Frédéric Ulrich, né à Hambourg en avril 1751, prédicateur réformé à Berlin». Or, Wolfgang Adam nous révèle que cette courte note de Payen, qui contient tout ce qu'on connaissait jusqu'à présent sur ce personnage mystérieux, est erronée, puisque Ulrich est né à Halle et non pas à Hambourg; cette affirmation suffit à elle seule à prouver l'étendue des découvertes et des nouveautés que cette étude propose. Ulrich fut bien plus qu'un simple «prédicateur réformé»: théologien, philosophe, historien, traducteur de textes de voyage, journaliste, il occupa sa courte existence (1751-1798) à la création d'un réseau important de relations sociales et intellectuelles. «Les publications d'Ulrich ont eu une grande résonance dans le panorama littéraire de son époque» (p. 105, traduit par nous-même. Nous remercions Maurizio Pirro de nous avoir aidée de manière savante et ponctuelle pour la compréhension de l'allemand). Un an à peine avant la traduction du texte de Montaigne, il traduisit et publia un autre texte de voyage en Suisse (p. 111). Wolfgang Adam consacre à la vaste bibliographie du traducteur une section de la bibliographie générale de l'œuvre (pp. 194-196). Selon le Dr. Payen, la traduction du Journal effectuée par Ulrich est un travail audacieux. Ulrich avait inséré dans le texte de Montaigne ses propres idées chaque fois que «cela lui a passé par la tête» (Z-Payen 432). Nous découvrons par cette étude que, au contraire, Ulrich fut un traducteur assez spécial, il est vrai, mais presque toujours correct et transparent lorsqu'il s'agissait de signaler son intervention. Le lecteur découvre par exemple qu'Ulrich ouvre son ouvrage par un Avant-propos du traducteur où il déclare très précisément qu'il va intervenir sur le texte. Il s'agit donc d'un choix de traduction bien précis. Adam affirme: «Ulrich prouve être un auteur qui a pleine conscience de ses choix et qui les soutient avec beaucoup de vigueur» (traduit par nous-même, p. 118). Quand, dans le texte, sa traduction est trop libre, non seulement il avoue son manque de fidélité au texte de départ dans une note, mais il y explique aussi pourquoi une 
traduction libre est meilleure qu'une traduction mot à mot (p. 124). Wolfgang Adam analyse, en les éclaircissant par des exemples, toutes les typologies d'intervention d'Ulrich, partant de celles où il adapte les idées de Montaigne à la philosophie des Lumières, pour arriver à celles où il insère dans le texte les commentaires de Querlon ou de Bartoli comme s'il s'agissait des mots de l'auteur. Le point de départ reste, tout de même, ce qu'Adam appelle la recherche d'une «Harmonie» (p. 146), d'une consonance avec le texte de Montaigne. Le dernier chapitre, le quatrième, présente un aperçu de la critique allemande et de la réception du texte du Journal en Allemagne du XVIII ${ }^{\mathrm{e}}$ au $\mathrm{xx}^{\mathrm{e}}$ siècle («Montaignes Journal de voyage: Kritik und Zustimmung (18. bis 20. Jahrhundert)», pp. 171-190). Là aussi nous découvrons des choses intéressantes sur la réception de la première traduction allemande du Journal de Montaigne. Comme, par exemple, le fait que le premier volume sorti en 1777 fut très mal accueilli par la critique et par les journaux et que par conséquent le deuxième, sorti en 1779, ne jouit d'aucune véritable attention. Quand, en 1808, une vingtaine d'années après la traduction d'Ulrich, un journaliste publie un compte-rendu du Journal de Montaigne (pp. 174-175), il traduit des extraits directement du français, puisqu'il ignore qu'il existe une traduction allemande (c'est ce qu'il avoue à la fin du texte, après qu'on lui a révélé l'existence de la traduction d'Ulrich). On n'a aucune certitude sur la connaissance, de la part de Goethe par exemple, du travail d'Ulrich. La traduction fut donc oubliée presque immédiatement après sa publication et la nouvelle traduction du Journal par Otto Flake, en 1908, ne fit que ratifier à jamais cet oubli. Il y a donc plus d'une raison pour lire ce livre de Wolfgang Adam qui présente une traduction mal connue et son traducteur, et qui semble avoir subi le même sort que celui du Journal de Montaigne en France après sa publication. Une riche bibliographie clôt ce volume, qui complète de manière admirable le panorama des études sur la réception du Journal. La clarté de la présentation, ainsi que la rigueur de l'analyse, en font une référence pour toutes les études sur le Journal de voyage. Une traduction française de ce volume pourrait contribuer à mettre à la disposition de la plupart des chercheurs et des spécialistes de Montaigne ces nouvelles informations. 